Bio - grafía. Escritos sobre la Biología y su Enseñanza. ISSN 2027-1034

Edición Extraordinaria. p.p. 1541 - 1547

Memorias del IX Encuentro Nacional de Experiencias en Enseñanza de la Biología y la

Educación Ambiental. IV Congreso Nacional de Investigación en Enseñanza de la

Biología.

\title{
TRABAJOS DE CAMPO Y DE LABORATORIO: DOS ESCENARIOS EN LA ENSEÑANZA DE LA BIOLOGÍA Y LA FORMACIÓN DOCENTE
}

\section{FIELD AND LABORATORY WORK: TWO SCENARIOS IN BIOLOGY TEACHING AND TEACHING TRAINING}

\author{
Nancy Fernández Marchesi ${ }^{1}$ \\ Elías Fco Amórtegui. ${ }^{2}$
}

\section{RESUMEN}

En este taller se realizará una reflexión sobre las ideas presentes en los docentes sobre las actividades de laboratorio y sobre las salidas de campo en la enseñanza de las ciencias naturales. Se pondrán en evidencia dichas concepciones a la luz de los marcos teóricos y los avances en las investigaciones de los autores de referencia. A partir de la confrontación de las concepciones con la literatura de la didáctica, se propone re escribir las propuestas desde un enfoque investigativo y contextualizado, desde la perspectiva del Conocimiento del Profesor. Al finalizar se espera poder socializar lo escrito entre los asistentes y así generar un espacio de comunidad fortaleciendo la dialéctica entre la teoría y la práctica. También otorgar al profesorado, algunas orientaciones sobre su planificación, su puesta en marcha y evaluación.

\section{ABSTRACT}

This workshop will reflect on the ideas present in teachers about laboratory activities and field trips in the teaching of natural sciences. These concepts will be exposed in the light of the theoretical frameworks and the advances in the investigations of the authors of reference. From the confrontation of the conceptions with the didactic literature, it is proposed to write the proposals from a research and contextualized approach, from the perspective of the Teacher's Knowledge. At the end, it is hoped to be able to socialize the writing between the attendees and thus generate a space of community strengthening the dialectic between theory and practice. Also give teachers some guidance on their planning, implementation and evaluation.

PALABRAS CLAVES: Trabajos Prácticos, Salidas de Campo, Prácticas de Laboratorio, Formación docente.

KEY WORDS: Practical Work, Field work, Laboratory Practices, Teacher Training.

\footnotetext{
${ }^{1}$ Instituto de Educación y Conocimiento. Universidad Nacional Tierra de Fuego.nfernandez@untdf.edu.ar

2 Universidad Surcolombiana. elias.amortegui@usco.edu.co
} 


\section{Bio - grafía. Escritos sobre la Biología y su Enseñanza. ISSN 2027-1034}

\section{Edición Extraordinaria. p.p. 1541 - 1547}

Memorias del IX Encuentro Nacional de Experiencias en Enseñanza de la Biología y la

Educación Ambiental. IV Congreso Nacional de Investigación en Enseñanza de la Biología.

\section{INTRODUCCIÓN}

La profesión docente se ha subestimado social y epistemológicamente al considerarse que para enseñar basta con tener dominio del conocimiento disciplinar en profundidad, dejando de lado el hecho de que como profesionales de la educación, los docentes deben poseer un conocimiento particular que, además de incluir el conocimiento del contenido, incluye aspectos didácticos, pedagógicos, entre otros, lo que les posibilita enseñar adecuadamente (Bromme, 1988; Valbuena, 2007; Tardif \& Lessard, 2014). Entre dichos conocimientos encontramos el Conocimiento Didáctico del Contenido, que incluye elementos tales como las finalidades de aprendizaje del alumnado, la evaluación de sus conocimientos, los contenidos de enseñanza, el conocimiento sobre el currículo, las dificultades de aprendizaje, el conocimiento escolar y en particular, las estrategias de enseñanza donde podemos ubicar el trabajo de campo y las actividades de laboratorio. (Amórtegui Cedeño \& Correa Sánchez, 2012; Park \& Chen, 2012; Valbuena Ussa, 2007)

Sumado a lo anterior, podría inferirse que ha habido poca reflexión sobre el trabajo de campo específicamente en la enseñanza de la Biología y la formación docente, generando así la necesidad de desarrollar propuestas formativas sobre el adecuado conocimiento de contenido y didáctico requerido para enseñar en el exterior de la escuela en ambientes naturales (Del Toro \& Morcillo, 2011), muestra de ello la poca literatura especializada en el contexto latinoamericano sobre la enseñanza de la Biología en el campo (Amórtegui Cedeño, Gavidia Catalán, \& Mayoral, 2016) y su casi inexistente aparición en la política educativa.

Del Toro (2014) argumenta la existente dificultad al momento de definir con claridad el concepto de actividad de campo, principalmente porque se suele asociar de manera directa con diversas actividades fuera del aula. Sin embargo, consideramos al igual que Morag \& Tal (2012) que las salidas al campo dentro de los ambientes de aprendizaje fuera de la escuela (educación formal), difiere de las visitas a museos, planetarios o centros de ciencia (educación no formal), dado que permite una experiencia directa con fenómenos naturales y la vida silvestre, además tiene una potencia altísima de generar comportamientos pro ambientales y conciencia sobre aspectos de la conservación, sobrepasando la idea de la educación centrada en el libro de texto.

Consideramos que las Prácticas de Campo adquieren un valor especial en la enseñanza y aprendizaje de la Biología pues permiten al alumnado abordar su objeto de estudio, "lo vivo", lo más cerca posible a sus condiciones naturales, con una perspectiva sistémica y holística que les permite comprender la relaciones que conforman el fenómeno viviente en conjunto con su ambiente: redes tróficas, adaptaciones, relaciones inter e intra específicas, biodiversidad, ecosistemas, autopoiesis, etc. Pero además ofrecen oportunidades educativas de alto valor relacionadas con aspectos procedimentales y actitudinales, como son la apreciación del significado de naturaleza, la valoración, conservación, disfrute sostenible de los recursos naturales, etc.

Una de las preguntas clave que nos hacemos al organizar nuestro Conocimiento Biológico es ¿Qué relaciones tienen los seres vivos entre sí y con su medio? Es evidente que los tópicos que debemos tratar para su respuesta son más fácilmente abordables a través de 


\section{Bio - grafía. Escritos sobre la Biología y su Enseñanza. ISSN 2027-1034}

\section{Edición Extraordinaria. p.p. 1541 - 1547}

Memorias del IX Encuentro Nacional de Experiencias en Enseñanza de la Biología y la

Educación Ambiental. IV Congreso Nacional de Investigación en Enseñanza de la Biología.

las Salidas de Campo, al conferir una perspectiva sistémica a lo vivo in situ difícilmente alcanzable sin las relaciones directas entre individuos y entornos.

Por su parte, las actividades prácticas de laboratorio se encuentran presente en todos los diseños curriculares, en las sugerencias didácticas, en los libros de texto, e incluso hay numerosas páginas web o canales de videos. Sin embargo, los objetivos educativos que tienen parecen no lograrse y, los profesores y profesoras optan por abandonar el eje conceptual de "lo experimental", porque no consiguen otorgarle sentidos epistemológicos claros y motivar a sus alumnos. Numerosos trabajos (de Pro Bueno, Sánchez Blanco, \& Valcárcel Pérez, 2008; Fernández Marchesi, 2014; Martínez Losada \& García Barros, 2003; Rivarosa \& Astudillo, 2013; Tenreiro-Vieira \& Marques Vieira, 2006), dan cuenta en sus análisis críticos, que las actividades de laboratorio, tanto las que diseñan los propios docentes como las propuestas por los libros de texto, siguen protocolos rígidos y estructurados tipo "receta" que promueven un escaso número de habilidades, y que no se condicen con los modelos de prácticas experimentales que se desarrollan en el ámbito del quehacer científico.

Podríamos asumir, que si los trabajos prácticos son actividades diseñadas para ser realizadas por los alumnos, con un grado variable de participación en su diseño y ejecución (del Carmen Martín, 2011), que buscan promover procesos cognitivos que faciliten la comprensión de un sistema explicativo sobre un hecho o fenómeno, que implica mucho más que la "simple observación y demostración"; entonces, ese conjunto de actividades prácticas que se hilvanan con una estrategia metódica particular (rigor y cualidad interpretativa), requiere desarrollar procesos y habilidades de alto valor cognitivo, que no se reducen a la manipulación de elementos y materiales de un laboratorio o campo.

Este enfoque de investigación implica entre otras cuestiones: problematizar la situación; promover el modelado de diseños posibles de interpretar y, jugar con la creatividad en la formulación; plantear preguntas que cuestionen el sentido del análisis de variables observables y teóricas; atreverse a validarlas en su resolución; atender al error experimental; analizar y cuestionar entre lo pensado-ejecutado-resultado; argumentar y ensayar respuestas y reflexionar sobre el proceso y su resultado. No caben dudas que el trabajo de laboratorio debe ser un componente fundamental de la enseñanza-aprendizaje de las ciencias.

Dicha actividad consiste en el uso de material de laboratorio para reproducir un fenómeno - para analizar una parte del mundo a estudiar, pudiéndose realizar tanto en un laboratorio como en cualquier aula (Leite \& Figueiroa, 2004). Son numerosos los motivos por los cuales introducir a las y los estudiantes en la realización de trabajos de laboratorio. Éstos poseen una gran potencialidad para abordar objetivos relacionados con:

- el aprendizaje de contenidos conceptuales y procedimentales,

- aspectos relacionados con la metodología científica,

- la promoción de capacidades de razonamiento como el pensamiento crítico y creativo, 


\section{Bio - grafía. Escritos sobre la Biología y su Enseñanza. ISSN 2027-1034}

\section{Edición Extraordinaria. p.p. 1541 - 1547}

Memorias del IX Encuentro Nacional de Experiencias en Enseñanza de la Biología y la

Educación Ambiental. IV Congreso Nacional de Investigación en Enseñanza de la Biología.

- el desarrollo de actitudes tales como la apertura de mente, objetividad y de desconfianza ante aquellos juicios de valor que carecen de evidencias necesarias.

Habitualmente los Trabajos de Laboratorio (TP) de laboratorio suelen presentarse como montajes ya elaborados para su simple manejo y/u observación, siguiendo guías tipo "receta de cocina". De este modo, la enseñanza centrada en la simple transmisión de conocimientos ya elaborados favorece y refuerza la concepción sobre la ciencia que sacraliza el trabajo experimental y que presenta al "Método Científico" como un conjunto de etapas correlativas, resaltando un tratamiento cuantitativo, un control riguroso, y olvidando y hasta rechazando todo lo que significa invención, creatividad, duda. Realizar trabajos prácticos como estrategia para superar la falta de interés por el aprendizaje de las ciencias cuenta con una larga tradición. Ahora bien, nos preguntamos: ¿Hasta qué punto las prácticas que se realizan, en mayor o menor número, contribuyen a dicha la familiarización con la ciencia?

Es importante responder a esto, a la luz de las concepciones de ciencias que trasmitimos en la enseñanza, porque cabría sospechar que el problema principal no es el número de TP realizados, sino la naturaleza de los mismos. Obviamente que cuestionamos ante todo el carácter de simple "receta", o el énfasis en la realización de mediciones y cálculos, con ausencia de aspectos fundamentales en la construcción del conocimiento científico. Discutir la relevancia del trabajo a realizar, el esclarecimiento de la problemática en que se inserta, la participación de las y los estudiantes en la construcción de la hipótesis y el diseño de los experimentos o el análisis de los resultados obtenidos, son cuestiones fundamentales a la hora de trasmitir una adecuada concepción de la actividad científica.

Este taller surge a partir de las tesis doctorales de los autores, por ello lo presentamos como una posibilidad de reflexión sobre la importancia tanto del trabajo de campo como el de laboratorio en el marco de la enseñanza y aprendizaje de la Biología; además se propone partir de la misma formación del profesorado y su práctica docente analizar sus fortalezas y en general abordar qué y cómo enseñar en el laboratorio y en el campo.

\section{OBJETIVOS DEL TALLER:}

1. Reflexionar sobre las salidas de campo y laboratorio.

2. Reconocer diversos tipos de experiencias, sus intencionalidades didácticas y sus posibles variantes

3. Analizar prácticas de campo y de laboratorio habituales en los libros de textos escolares y las prácticas educativas.

4. Analizar los tipos de trabajos prácticos de laboratorio que enuncia la Didáctica de las Ciencias Experimentales

5. Establecer algunos elementos sobre la planificación de experiencias áulicas de laboratorio y las salidas de campo

6. Diseñar experiencias sencillas en la diversidad de contextos y niveles de enseñanza de los participantes 
Bio - grafía. Escritos sobre la Biología y su Enseñanza. ISSN 2027-1034

Edición Extraordinaria. p.p. 1541 - 1547

Memorias del IX Encuentro Nacional de Experiencias en Enseñanza de la Biología y la

Educación Ambiental. IV Congreso Nacional de Investigación en Enseñanza de la

Biología.

\section{ASPECTOS PROCEDIMENTALES}

Actividad 1. ¿Qué pensamos sobre las salidas de campo y los trabajos de laboratorio?

En el campo de la Didáctica de las Ciencias es ampliamente reconocido que las Prácticas de Campo, al igual que los laboratorios, simulaciones, entre otros, son considerados como Trabajos Prácticos (Caamaño, 2003; del Carmen Martín et al., 2000), y que además suponen la articulación de diferentes tipos de actividades, mediante un enfoque integrado, en el que la teoría y la práctica se entrelazan en un tratamiento conjunto. Para comenzar la actividad, el profesorado asistente debe organizarse en cinco grupos y enumerarse. De acuerdo al número del grupo, cada uno debe responder en un respectivo formato la pregunta que le corresponde de manera individual.

Grupo 1: ¿Qué es una Práctica de Campo? ¿Qué es una actividad práctica de laboratorio?

Grupo 2: ¿Qué Relaciones entre la teoría y la práctica se dan en una práctica de laboratorio?:

Grupo 3: ¿Cuáles son las finalidades de realizar una salida de campo?:

Grupo 4: ¿Cuáles son los elementos que debería contener la Guía de una práctica de laboratorio?:

Grupo 5: ¿Cuáles son los aportes de las prácticas de campo y de las actividades de laboratorio a la formación de docentes de ciencias naturales?

Actividad 2. ¿Cómo representamos nuestras ideas sobre las Prácticas de Campo y las actividades de laboratorio?

En muchas ocasiones, los dibujos y esquemas nos ayudan a expresar y a comprender nuestras ideas. Por ello, vamos a desarrollar esta técnica en la expresión de nuestras ideas. Primero discutir en cada grupo las respuestas individuales expresadas en la actividad anterior y consignar el consenso en el respectivo formato. Luego, realizar de manera conjunta un dibujo en un cartel que exprese dicho consenso. Recuerda que no se debe incluir ningún texto en los dibujos y todos deben participar en la elaboración del mismo. Colocar en lugares visibles los dibujos realizados y que cada grupo interprete los carteles elaborados. Discutir a partir de la orientación de los profesores talleristas.

Actividad 3. ¿Qué recordamos sobre las salidas de campo y los trabajos de de laboratorio que realizamos cuando estábamos en la universidad?

Cada grupo de trabajo discute sobre una actividad de campo o laboratorio que recuerden de la época en la que eran estudiantes universitarios. Bajo un consenso general, describen dicha actividad teniendo en cuenta el respectivo formato. 
Bio - grafía. Escritos sobre la Biología y su Enseñanza. ISSN 2027-1034

\section{Edición Extraordinaria. p.p. 1541 - 1547}

Memorias del IX Encuentro Nacional de Experiencias en Enseñanza de la Biología y la

Educación Ambiental. IV Congreso Nacional de Investigación en Enseñanza de la Biología.

\section{Actividad 4: ¿Qué podemos tener en cuenta para planificar y desarrollar trabajos de campo y laboratorio?}

A partir de la exposición de los talleristas y la lectura de un texto disparador sobre la didáctica de las actividades prácticas de laboratorio y de campo, se les propondrá a los asistentes redactar una guía teniendo en cuenta el marco teórico desarrollado. La propuesta deberá incluir: actividades previas, durante y posteriores a la actividad de campo o de laboratorio. El diseño se realizará en grupo y tendrá los siguientes criterios para su elaboración: Nivel de escolaridad, contextualización, contenido a enseñar, enfoque investigador.

\section{Actividad 5: Socializando nuestras propuestas}

Cada grupo socializará al resto la propuesta elaborada. De ser posible, se realizarán en soporte digital para que pueda ser compartida con el resto de los asistentes al taller.

\section{ESTRATEGIAS DE EVALUACIÓN}

Asumimos aquí que el profesorado asistente al taller, con base en la reflexión y trabajo realizado, pueda acercarse a la elaboración grupal de un Trabajo Práctico de laboratorio o una actividad de campo, adecuándose a los criterios tratados en el taller. Para ellos se les solicitará que completen un cuadro en el cual puedan expresar qué creian antes del taller y que piensan luego del taller sobre las actividades prácticas de laboratorio y las actividades prácticas de campo.

\section{REFERENCIAS}

Amórtegui Cedeño, E., \& Correa Sánchez, M. (2012). Las prácticas de campo planificadas en el proyecto curricular de licenciatura en biología, Universidad Pedagógica Nacional: caracterización desde la perspectiva del conocimiento profesional del profesor de biología. Universidad Pedagógica Nacional.

Amórtegui Cedeño, E., Gavidia Catalán, V., \& Mayoral, O. (2016). Las prácticas de campo en la enseñanza de la biología y la formación docente: estado actual de conocimiento. TED: Tecne, Episteme y Didaxis, Extraordin, 9-15.

Caamaño, A. (2003). Los trabajos prácticos en ciencias. En M. P. Jiménez Aleixandre (Ed.), Enseñar Ciencias (pp. 95-118). Barcelona: Graó.

de Pro Bueno, A., Sánchez Blanco, G., \& Valcárcel Pérez, M. (2008). Análisis de los libros de texto de Física y Química en el contexto de la Reforma LOGSE. Enseñanza de las Ciencias, 26(2), 193-210.

del Carmen Martín, L. (2011). El lugar de los trabajos prácticos en la construcción del conocimiento científico en la enseñanza de la biología y la geología. En P. Cañal (Ed.), Didáctica de la biología y la geología (pp. 91-108). Barcelona: Graó.

del Carmen Martín, L., Caballer, M., Furió, C., Gómez Crespo, M., Jiménez Aleixandre, M., Jorba, J., ... Vilches, A. (2000). La enseñanza y el aprendizaje de las ciencias de la naturaleza en la educacion secundaria. Barcelona: Horsori.

Del Toro, R. (2014). Concepciones y prácticas del profesorado acerca de las actividades 
Bio - grafía. Escritos sobre la Biología y su Enseñanza. ISSN 2027-1034

\section{Edición Extraordinaria. p.p. 1541 - 1547}

Memorias del IX Encuentro Nacional de Experiencias en Enseñanza de la Biología y la

Educación Ambiental. IV Congreso Nacional de Investigación en Enseñanza de la Biología.

de campo en educación secundaria de Biología en diferentes contextos educativos: los casos de Dinamarca, Campiñas (Sao Paulo, Brasil) y la comunidad de Madrid. Universidad Complutense de Madrid.

Del Toro, R., \& Morcillo, J. G. (2011). Las actividades de campo en Educación Secundaria. Un estudio comparativo entre Dinamarca y España. Enseñanza de las Ciencias de la Tierra, 19(1), 39.

Fernández Marchesi, N. (2014). Los trabajos prácticos de laboratorio de Biología en los libros de texto de Ciencias Naturales para el Nivel Secundario utilizados en la Ciudad de Ushuaia. Tesis de Maestría. Facultad de Ingeniería. Universidad Nacional del Centro de la Provincia de Buenos Aires.

Leite, L., \& Figueiroa, A. (2004). Las actividades de laboratorio y la explicación científica en los manuales escolares de ciencias. Alambique. Didáctica de las ciencias experimentales, 10(39), 20-30.

Martínez Losada, C., \& García Barros, S. (2003). Las actividades de primaria y ESO incluidas en libros escolares: ¿Qué objetivo persiguen? ¿Qué procedimientos enseñan? Enseñanza de las Ciencias, 21, 243-264.

Morag, O., \& Tal, T. (2012). Assessing learning in the outdoors with the field trip in natural environments (FiNE) framework. International Journal of Science Education, 34(5), 745-777.

Park, S., \& Chen, Y.-C. (2012). Mapping out the integration of the components of pedagogical content knowledge (PCK): Examples from high school biology classrooms. Journal of Research in Science Teaching, 49(7), 922-941.

Rivarosa, A., \& Astudillo, C. (2013). Las prácticas científicas y la cultura: una reflexión necesaria para un educador de ciencias. Revista CTS, 8(23), 45-66.

Tenreiro-Vieira, C., \& Marques Vieira, R. (2006). Diseño y validación de actividades de laboratorio para promover el pensamiento crítico de los alumnos. Revista Eureka sobre Enseñanza y Divulgación de las Ciencias, 3(3), 452-466.

Valbuena Ussa, E. (2007). El conocimiento didáctico del contenido biológico: estudio de las concepciones disciplinares y didácticas de futuros docentes de la Universidad Pedagógica Nacional (Colombia). Tesis doctoral. Universidad Complutense de Madrid. 